\title{
Improving, Restoring, and Managing Natural Resources in Florida: Sources of Technical Assistance for Urban Homeowners 1
}

Mark Hostetler, Martin Main, Chris Demers ${ }^{2}$

\section{Introduction}

Over the last decade approximately 100,000 new single-family, detached homes have been built annually in Florida, making it one of the most active areas of new residential community development in the United States. In the face of this rapid growth, many Florida communities are looking for support in how to preserve (and even enhance) their local quality of life. Collectively, urban communities can greatly affect our natural resources.

Decisions made by each homeowner interact in unique ways to affect the use and conservation of water, energy, and our plants and animals. The quantity and quality of water, wildlife habitat, and energy are closely tied to one another. For example, planting several native trees near a house can have several positive impacts. First, native trees provide food for wildlife. Second, they provide shade for the house to conserve energy. Plus, they conserve water because native trees are adapted to the local climate and do not need much watering. As a result, a homeowner saves time and money because he or she is not using as much water or energy. If each homeowner plants native trees, more local water and energy resources are conserved.

In fact, the way people design and manage their homes, yards, and neighborhoods can impact surrounding natural areas. For example, invasive exotic plants in individual yards can spread to surrounding natural areas. Also, watering lawns can cause the water table to draw down, drying up nearby wetlands.

The purpose of this document is to summarize sources of information from federal, state, and non-governmental organizations that provide technical assistance to urban landowners interested in conserving natural resources (Table 1). Additional information regarding natural resource conservation on properties is available on the University of Florida IFAS, Cooperative Extension Electronic Data Information Source (EDIS http://edis.ifas.ufl.edu/). Some highlighted EDIS

1. This document is WEC-193, one of a series of the Wildlife Ecology and Conservation Department, Florida Cooperative Extension Service, Institute of Food and Agricultural Sciences, University of Florida. Original publication date November 2004. Visit the EDIS Web Site at http://edis.ifas.ufl.edu for more publications.

2. Mark E. Hostetler, Assistant Professor and Martin B. Main, Associate Professor, Department of Wildlife Ecology \& Conservation, Florida Cooperative Extension Service, Institute of Food and Agricultural Sciences, University of Florida, Gainesville, 32611.

Chris Demers, Forest Stewardship Coordinator, School of Forest Resources and Conservation, Cooperative Extension Service, Institute of Food and Agricultural Sciences, University of Florida, Gainesville, FL 32611.

The Institute of Food and Agricultural Sciences (IFAS) is an Equal Employment Opportunity - Affirmative Action Employer authorized to provide research, educational information and other services only to individuals and institutions that function without regard to race, creed, color, religion, age, disability, sex, sexual orientation, marital status, national origin, political opinions or affiliations. For information on obtaining other extension publications, contact your county Cooperative Extension Service office. Florida Cooperative Extension Service / Institute of Food and Agricultural Sciences / University of Florida / Larry R. Arrington, Interim Dean 
documents are provided below:

1. Tips on landscaping for wildlife:

http://edis.ifas.ufl.edu/

TOPIC_Landscaping_for_Wildlife

2. Energy conservation tips:

http://edis.ifas.ufl.edu/TOPIC_Saving_Energy

3. Environmental landscaping:

http://edis.ifas.ufl.edu/TOPIC_Enviroscaping

4. Water conservation in the yard:

http://edis.ifas.ufl.edu/

TOPIC_Lawns_and_Water_Conservation

5. Overall environmentally friendly living:

http://edis.ifas.ufl.edu/

TOPIC_Environmentally_Friendly_Living

6. Water conservation tips:

http://edis.ifas.ufl.edu/

TOPIC_Lawns_and_Water_Conservation

7. Conservation options (easements, etc.) for private landowners:

http://edis.ifas.ufl.edu/UW194

8. Evaluating private lands for conservation:

http://edis.ifas.ufl.edu/UW195 
Table 1. Organizations and programs available to urban communities to provide technical assistance for conservation of natural resources.

\begin{tabular}{|c|c|c|}
\hline ORGANIZATION & CONTACT INFORMATION & COMMENTS \\
\hline \multicolumn{3}{|l|}{ Federal } \\
\hline \multirow[t]{5}{*}{$\begin{array}{l}\text { Environmental } \\
\text { Protection Agency }\end{array}$} & $\begin{array}{l}\text { Energy Star } \\
\text { http://www.energystar.gov/ }\end{array}$ & $\begin{array}{l}\text { Assistance to property owners to } \\
\text { conserve energy within a building }\end{array}$ \\
\hline & $\begin{array}{l}\text { Office of Pollution and } \\
\text { Prevention, Community } \\
\text { Assistance Programs } \\
\text { http://www.epa.gov/opptintr/cahp/ }\end{array}$ & $\begin{array}{l}\text { An educational resource to find out about } \\
\text { environmental facts concerning land, air, } \\
\text { and water }\end{array}$ \\
\hline & $\begin{array}{l}\text { Envirofacts } \\
\text { http://www.epa.gov/enviro/ }\end{array}$ & $\begin{array}{l}\text { An educational resource to find out about } \\
\text { environmental facts concerning land, air, } \\
\text { and water }\end{array}$ \\
\hline & $\begin{array}{l}\text { Water use efficiency program } \\
\text { http://www.epa.gov/owm/water- } \\
\text { efficiency/index.htm }\end{array}$ & $\begin{array}{l}\text { Information on water efficient tools and } \\
\text { management strategies }\end{array}$ \\
\hline & $\begin{array}{l}\text { Reduce solid waste } \\
\text { http://www.epa.gov/epaoswer/ } \\
\text { non-hw/reduce/catbook/ } \\
\text { index.htm }\end{array}$ & $\begin{array}{l}\text { Tips on simple ways to reduce the } \\
\text { amount of solid waste }\end{array}$ \\
\hline $\begin{array}{l}\text { U.S. Department. of } \\
\text { Agriculture }\end{array}$ & $\begin{array}{l}\text { Natural Resources Conservation } \\
\text { Service (NRCS) } \\
\text { http://www.nrcs.usda.gov/ }\end{array}$ & $\begin{array}{l}\text { Multiple programs that provide technical } \\
\text { and financial support to assist } \\
\text { homeowners and communities to } \\
\text { conserve natural resources }\end{array}$ \\
\hline \multicolumn{3}{|l|}{ State } \\
\hline \multirow[t]{3}{*}{$\begin{array}{l}\text { University of Florida } \\
\text { IFAS }\end{array}$} & $\begin{array}{l}\text { Cooperative Extension Electronic } \\
\text { Data Information Source } \\
\text { http://edis.ifas.ufl.edu/ }\end{array}$ & $\begin{array}{l}\text { Searchable database for technical } \\
\text { information on many topics related to } \\
\text { management of natural resources }\end{array}$ \\
\hline & $\begin{array}{l}\text { Department of Wildlife Ecology \& } \\
\text { Conservation - Backyard } \\
\text { landscaping for wildlife } \\
\text { http://www.wec.ufl.edu/extension/ } \\
\text { landscaping.htm }\end{array}$ & $\begin{array}{l}\text { Information and program that help } \\
\text { property owners evaluate their landscape } \\
\text { for Florida wildlife }\end{array}$ \\
\hline & $\begin{array}{l}\text { Florida Yards \& Neighborhoods } \\
\text { http://hort.ufl.edu/fyn/ }\end{array}$ & $\begin{array}{l}\text { Helps residents to establish } \\
\text { environmentally friendly yards }\end{array}$ \\
\hline $\begin{array}{l}\text { Florida State } \\
\text { University }\end{array}$ & $\begin{array}{l}\text { Florida Natural Areas Inventory } \\
\text { http://www.fnai.org }\end{array}$ & $\begin{array}{l}\text { Information about conserving Florida's } \\
\text { biodiversity }\end{array}$ \\
\hline \multicolumn{3}{|l|}{ Non-governmental } \\
\hline $\begin{array}{l}\text { National Wildlife } \\
\text { Federation }\end{array}$ & $\begin{array}{l}\text { NWF Backyard Wildlife Program: } \\
\text { http://www.nwf.org/ } \\
\text { backyardwildlifehabitat/ }\end{array}$ & $\begin{array}{l}\text { Has information about ways to design a } \\
\text { wildlife-friendly yard and also a } \\
\text { certification program }\end{array}$ \\
\hline
\end{tabular}


Improving, Restoring, and Managing Natural Resources in Florida: Sources of Technical....

Table 1. Organizations and programs available to urban communities to provide technical assistance for conservation of natural resources.

\begin{tabular}{||l|l|l||}
\hline \hline \multicolumn{1}{|c|}{ ORGANIZATION } & \multicolumn{1}{|c||}{ CONTACT INFORMATION } & \multicolumn{1}{|c|}{ COMMENTS } \\
\hline Humane Society & $\begin{array}{l}\text { Humane Society's urban wildlife } \\
\text { program: } \\
\text { http://www.hsus.org/ace/19400 }\end{array}$ & $\begin{array}{l}\text { Has information about ways to design a } \\
\text { wildlife-friendly yard and also a } \\
\text { certification program }\end{array}$ \\
\hline $\begin{array}{l}\text { Florida Native Plant } \\
\text { Society }\end{array}$ & $\mathrm{http}: / / \mathrm{www}$. fnps.org/ & $\begin{array}{l}\text { Lists of native plants, tips on using native } \\
\text { plants in your landscape, and locations of } \\
\text { native plant nurseries }\end{array}$ \\
\hline $\begin{array}{l}\text { Florida Exotic Plant } \\
\text { Council }\end{array}$ & $\mathrm{http://www.fleppc.org/}$ & $\begin{array}{l}\text { Information, programs, and identification } \\
\text { tips on how to identify and eradicate } \\
\text { Florida exotic invasive plants }\end{array}$ \\
\hline \hline
\end{tabular}

